\title{
Erratum to: Is aclidinium alone or combined with a $L A B A$ a rational choice for symptomatic COPD patients?
}

\author{
F. Blasi ${ }^{*}$, G. W. Canonica ${ }^{2}$ and M. Miravitlles ${ }^{3}$
}

\section{Erratum}

In the original publication of this article [1], the funding section omitted the following information: This work was funded by Momento Medico srl, which has also contributed to the costs for the literature selection and editorial assistance.

\footnotetext{
Author details

'Department of Pathophysiology and Transplantation, Università degli Studi di Milano, Cardio-thoracic unit and Cystic Fibrosis Adult Center Fondazione IRCCS Cà Granda Ospedale Maggiore Policlinico Milano, Milan, Italy.

${ }^{2}$ Department of Biomedical Science, Personalized Medicine Clinic: Asthma \& Allergy - Humanitas Clinical and Research Center, Humanitas University -

Rozzano (Milano), Milan, Italy. ${ }^{3}$ Pneumology Department, Hospital Universitari Vall d'Hebron, Barcelona, Spain.
}

Received: 26 January 2017 Accepted: 31 January 2017

Published online: 10 February 2017

\section{References}

1. Blasi F, et al. Is aclidinium alone or combined with a LABA a rational choice for symptomatic COPD patients? Respir Res. 2017;18:19. doi:10.1186/s12931017-0506-0.

\footnotetext{
*Correspondence: francesco.blasi@unimi.it

${ }^{1}$ Department of Pathophysiology and Transplantation, Università degli Studi di Milano, Cardio-thoracic unit and Cystic Fibrosis Adult Center Fondazione IRCCS Cà Granda Ospedale Maggiore Policlinico Milano, Milan, Italy
} 